\title{
Sull'interpretazione del patto delle nazioni unite sui diritti economici, sociali e culturali: regole, criteri ermeneutici e comparazioni*
}

\section{Interpretation of the United Nations Covenant on Economic, Social and Cultural Rights: Rules, Hermeneutical Criteria and Comparisons}

Francesco Seatzu**

Sommario: I. Introduzione. II. L'interpretazione del Patto sui diritti economici, sociali e culturali da parte del Comitato nella teoria. III. L'interpretazione del Patto sui diritti economici, sociali e culturali da parte del Comitato nella prassi.

* Artículo recibido el 6 de mayo de 2011 y aprobado para publicación el 28 de septiembre de 2011.

Il testo, con l'opportuno corredo di note a piè di pagina ed alcuni aggiornamenti e integrazioni, riproduce la comunicazione presentata dall' Autore alla giornata di studi del 29 aprile 2010 sul tema: "L'efficacia della Convenzione europea per la salvaguardia dei diritti dell'uomo nell'ordinamento interno", tenutasi presso la Scuola di Studi Superiori “Giacomo Leopardi” dell'Università degli studi di Macerata.

** Professore Straordinario di Diritto Internazionale e dell'Unione europea, Università degli studi di Cagliari. 
Resumen: El Pacto Internacional de Derechos Económicos, Sociales y Culturales ("Pacto") confiere al Comité de la ONU sobre Derechos Económicos, Sociales y Culturales (“CDESC”) una jurisdicción muy amplia para dar una interpretación no vinculante del propio Pacto. El Comité DESC ha utilizado dos herramientas principales para dilucidar los diferentes tipos de obligaciones en virtud del Pacto: informes de los Estados y las observaciones generales. Desde su séptimo período de sesiones, en 1992, el CDESC empezó a adoptar las observaciones finales sobre los informes presentados por el Estado. Esta práctica permite que el Comité proporcione un análisis más específico de actuación de los Estados, el grado de su cumplimiento, y también identifica las formas de violaciones de los derechos económicos, sociales y culturales ("DESC") y ofrece recomendaciones detalladas sobre las posibles maneras de proteger los derechos con ejemplos concretos. La práctica extensa del CDESC ha contribuido a una interpretación extensiva de los DESC, que se pueden aplicar a todos ellos. La segunda herramienta a disposición del CDESC es la publicación de las observaciones generales y declaraciones.

Palabras clave: Pacto Internacional de Derechos Económicos, Sociales y Culturales; Comité de Derechos Económicos, Sociales y Culturales; Convención de Viena de 1969 sobre Derecho de los Tratados.

ABSTRACT: The International Covenant on Economic, Social and Cultural Rights ("ICESCR") confers on the UN Committee on Economic, Social and Cultural Rights ("CESCR") a very broad jurisdiction to give a non-binding interpretation of the Covenant itself. The CESCR has used two main tools to elucidate the different types of obligations under the ICESCR, States Reports and General Comments. Since its seventh session, in 1992, the CESCR started adopting Concluding Observations regarding submitted State reports. This practice allows the Committee to provide more specific analysis of States' performan$c e$, the degree of their accomplishment, but also identifies forms of violations of economic, social and cultural rights ("ESCR") and gives detailed recommendations concerning the possible ways of protecting rights with specific examples. The extensive practice of the CESCR has therefore contributed to a wide interpretation of ESCR that may be applied to all ESCR. The second tool available to the CESCR is the publication of General Comments and Statements.

Descriptors: International Covenant on Economic, Social and Cultural Rights; UN Committee on Economic, Social and Cultural Rights; The 1969 Vienna Convention on the Law of Treaties.

RÉSUMÉ: Le Pacte International relatif aux Droits Economiques, Sociaux et Culturels («Pacte») donne au Comité des Nations Unies relatif aux droits économiques, sociaux et culturels ("CDESC») une compétence très vaste pour donner une interprétation pas obligatoire du Pacte lui-même. Le CDESC a utilisé deux outils principaux pour élucider les différents types d'obligations selon le Pacte: Des rapports des États et les observations généraux. Depuis sa septième étape des sessions en 1992, le CDESC a commencé à adopter les observations finales sur les rapports présentés par l'État. Cette pratique permet au Comité de fournir une analyse plus spécifique de la performance des États, le degré de conformité, et aussi identifier les formes de violations des droits économiques, sociaux et culturels ( $\mathrm{DESC}$ ) et fournit des recommandations détaillées sur d'éventuelles façons de protéger les droits avec des exemples spécifiques. La pratique normale du CDESC a contribué à une interprétation plus grande des DESC qui peut être appliqués. Le deuxième outil disponible pour le CDESC est la publication d'observations générales et déclarations.

Mots-clés: Pacte international relatif aux Droits Economiques, Sociaux et Culturels, Comité des Nations unies relatif aux Droits Economiques, Sociaux et Culturels; la Convention de 1969 sur le droit des traités. 


\section{INTRODUZIONE}

\section{Premessa}

Esistono, a nostro avviso, almeno tre approcci alternativi allo studio della "giurisprudenza" del Comitato delle Nazioni Unite sui diritti economici, sociali e culturali (il "Comitato" o "CESCR"). Il primo di essi consiste nella valutazione dell'impatto delle Osservazioni Generali (“General Comments”) e delle Osservazioni Finali ("Concluding Observations”) negli ordinamenti giuridici degli Stati contraenti. Un secondo approccio di tipo più internazionalistico consiste, invece, nell'esame delle tecniche e dei criteri ermeneutici impiegati dal Comitato nell'elaborazione dei sopra richiamati atti. Esiste, infine, un approccio di tipo eminentemente sociologico, che si traduce nella considerazione delle questioni connesse all'attuazione o più esattamente all'osservanza dei singoli "General Comments" e delle "General Observations" negli Stati contraenti del Patto sui diritti economici, sociali e culturali (il "Patto"). In questa sede si intende adottare la seconda prospettiva sopra indicata e quindi ci si soffermerà sull'interpretazione del Patto da parte del Comitato. Si avrà riguardo alla "giurisprudenza" del CESCR in una prospettiva di occasionale confronto con le corrispondenti prassi di altri organismi di garanzia (giurisdizionali e quasi giurisdizionali) come, ad esempio, la Corte europea dei diritti dell'uomo nonché con le prassi dei meccanismi di controllo operanti presso le principali organizzazioni

1 Il Patto, adottato dall'Assemblea Generale delle Nazioni Unite il 16 dicembre 1966, è entrato in vigore il 3 gennaio 1976. Il testo in italiano del Patto è riprodotto anche in Luzzatto, Riccardo, Pocar Fausto, Codice di diritto internazionale pubblico, Italia, Torino-Giappichelli editore, 2010, pp. 175 y ss. Sul Patto, senza alcuna pretesa di completezza, si vedano i recenti lavori di Ssenyonjo, Manisuli, Economic, Social and Cultural Rights in International Law, United Kingdom, Oxford-Hart Publishing, 2009; Bestagno, Francesco (a cura di), I diritti economici, sociali e culturali: Promozione e tutela nella comunità internazionale, Italia, Milano-Giuffré editore, 2009; Pineschi, Laura, "Il Patto delle Nazioni Unite sui diritti economici, sociali e culturali", in Pineschi, Laura (a cura di), La tutela internazionale dei diritti umani: Norme, garanzie, prassi, Italia, Milano-Giuffré editore, 2006, pp. 129 y ss. V. anche l'ormai classico manuale di Craven, Matthew The International Covenant on Economic, Social and Cultural Rights: A Perspective on its Development, United Kingdom, Oxford-OUP, 1995. 
internazionali a carattere finanziario (questi ultimi, più di altri sistemi internazionali di controllo, fortemente condizionati nella loro operatività dalle libertà e dai diritti socio-economici e culturali contenuti nel Patto). Non ci si soffermerà invece sulla rilevanza pratica del predetto approccio alla "giurisprudenza" del Comitato, perché di palmare evidenza e quindi superflua. In argomento, è sufficiente l'osservazione che soltanto mediante un'appropriata considerazione delle regole e dei criteri ermeneutici utilizzati dal Comitato è possibile ricavare gli orientamenti generali nonché riconoscere le eventuali peculiarità della "costituzione materiale" del Patto. ${ }^{2}$

L'esame della predetta "giurisprudenza" verrà realizzato avendo riguardo agli orientamenti della prassi che, almeno a nostro parere, sembrano maggiormente significativi perché esemplificativi delle tecniche interpretative impiegate. Ancora, lo studio della materia sarà affrontato in una duplice prospettiva. Innanzitutto, la "giurisprudenza" del Comitato sarà considerata dando risalto alle modalità con cui questo organismo esercita la sua funzione di interpretazione del diritto (da intendersi come l'insieme delle disposizioni e dei precetti contenuti nel Patto e nel Protocollo facoltativo al Patto sui diritti economici, sociali e culturali che prevede una procedura di comunicazione individuale). ${ }^{3}$ Succes-

2 Sul punto v. Odello, Marco, Seatzu, Francesco, Ensuring and Enforcing Economic, Social and Cultural Rights: The Jurisprudence of the UN Committee on Economic, Social and Cultural Rights, United Kingdom, London, Routledge, 2011 (in corso di pubblicazione), Cap. III.

3 Il Protocollo, adottato con risoluzione 63/117dell'Assemblea Generale delle Nazioni Unite il 10 dicembre 2008, non è ancora entrato in vigore. Per il testo del Protocollo in lingua originale (inglese) v. http://www2.ohchr.org/english/law/docs/A.RES.63.117_en pdf Sul Protocollo facoltativo v. tra i molti Gargiulo, Pietro, "Il protocollo facoltativo al patto sui diritti economici, sociali e culturali”, in Venturini, Gabriella, Bariatti, Stefania (a cura di), Diritti individuali e giustizia internazionale: Liber Fausto Pocar, t. I, Italia, Milano-Giuffré editore, 2009, p. 339 ss; Coomans, Fons, "Het nieuwe facultatieve klachtrecht Protocol bij het Internationaal Verdrag inzake Economische, Sociale en Culturele Rechten, in Nederlands tijdschrift voor de mensenrechten", NCJM-Bulletin, 2010, p. 263 ss.; De Schutter, Olivier, "Le Protocole facultatif au Pacte international sur les droits économiques, sociale set culturelles", CRIDHO Working Paper, 2005, www.fidh.org/IMG/pdf/PIDESC Protocole.pdf, 2005; Vandenbogaerde, Arne, Vandenhole, Wouter, "The Optional Protocol to the International Covenant on Economic, Social and Cultural Rights: an Ex Ante Assessment of its Effectiveness in Light of the Drafting Process”, Human Rights Law Review, 2010, pp. 207 y ss.; Langa, Pius, “Taking Dignity Seriously: Judicial Reflections on the Optional Protocol to the ICESCR", Nordisk tidsskrift for mennenskerettigheter, 2009, pp. 29 y ss. 
sivamente si passerà ad una trattazione delle modalità applicative delle norme pattizie nella teoria e nella pratica.

\section{Cenni alla struttura e al funzionamento del Comitato dei diritti economici, sociali e culturali}

Alcuni cenni forzatamente brevi devono farsi, innanzitutto, alla struttura istituzionale e all'operatività del Comitato. Istituito come organo di garanzia dalla risoluzione ECOSOC 1985/17 del 28 maggio 1985 e composto da 18 esperti indipendenti - designati dagli Stati contraenti a titolo individuale ed in considerazione delle loro competenze nel campo dei diritti economici, sociali e culturali per un mandato (rinnovabile) di quattro anni- — ${ }^{4}$ il Comitato è attualmente provvisto di talune competenze comuni alla maggioranza degli altri comitati (cd."treaty bodies") incaricati di supervisionare l'applicazione delle principali convenzioni delle Nazioni Unite sui diritti dell'uomo5 ${ }^{5}$ In sintesi, può ricordarsi che i componenti del Comitato vengono eletti sulla base di un'equa ripartizione geografica e, a questo fine, gli Stati membri delle Nazioni Unite si trovano ripartiti in cinque gruppi geografici: Europa dell' Ovest e altri; Europa dell'Est; Africa; Asia e Americhe. ${ }^{6}$

Soffermandoci adesso sulle funzioni e le procedure del Comitato una bi-partizione deve essere effettuata tra le procedure non contenziose, da un lato, e le procedure contenziose dall'altro lato. Nell' ambito delle procedure non contenziose il Comitato è incaricato di accertare il rispetto degli obblighi convenzionali degli Stati nell'assenza di una controversia tra un Stato e un altro Stato oppure tra un individuo e lo Stato alla cui giurisdizione questi si trova sottoposto. La procedura non con-

4 Cfr. Pineschi, Laura, "Il Patto delle Nazioni Unite sui diritti economici, sociali e culturali”, in Pineschi, Laura (a cura di), La tutela internazionale, cit., p. 142, n. 48, la quale ricorda che ciascun membro prima di assumere l'incarico deve dichiarare solennemente di esercitare le proprie funzioni in modo imparziale.

5 Sul CESCR, oltre al nostro lavoro citato supra n. 2, si rinvia a Malinverni, Giorgio,"Il Comitato dei diritti economici, sociali e culturali”, in Bestagno, Francesco (a cura di), I diritti economici, sociali e culturali, cit., pp. 188 y ss.

6 V., oltre ai contributi citati supra n. 3, Gherari, Habib, "Le Comite des Droits Economiques, Sociaux et Culturels”, RGDIP, 1992, pp. 80 y ss. 
tenziosa più rilevante è quella del rapporto, istituita dall'obbligo pattizio per gli Stati contraenti di sottoporre delle relazioni periodiche (una ogni cinque anni) successivamente alla proposizione di un loro rapporto iniziale ${ }^{7}$. Oggetto di siffatti rapporti periodici è lo stato di attuazione dei diritti socio-economici e culturali nell' ordinamento giuridico interno dello Stato autore del rapporto. Ciò al fine di garantire un'effettiva attuazione dei diritti socio-economici a livello statale - in particolare la rimozione dei principali ostacoli all'attività delle organizzazioni non governative nella promozione dei diritti e delle libertà in campo socio-economico - anche tramite l'instaurazione o il potenziamento della cooperazione tra i singoli Stati contraenti. I General Comments finora adottati, ventuno in numero, costituiscono uno dei principali strumenti interpretativi del Patto forniti da e utilizzati dallo stesso CESCR. Vanno altresì menzionate, sempre nel contesto delle procedure non contenziose, le cosiddette "procedure speciali", istitutive di relatori speciali, impiegate sia per affrontare un particolare tema sempre connesso all' attuazione dei diritti socio-economici e culturali sia le questioni eventualmente poste da un determinato Stato contraente nell'attuazione al proprio interno di siffatti diritti fondamentali.

Quanto alle procedure contenziose - le quali presuppongono, invece, l'esistenza di una controversia tra due Stati o tra un individuo e uno Stato - il CESCR, diversamente da altri comitati delle Nazioni Unite sui diritti umani, non è competente ad esaminare né ricorsi interstatali né ricorsi individuali ${ }^{8}$. Contrariamente all'obbligo di redigere rapporti periodici che sorge, direttamente ed esclusivamente, dalla ratifica del Patto quello che impone agli Stati contraenti di accettare dei ricorsi individuali richiede il loro esplicito consenso mediante la ratifica del Protocollo al Patto sui diritti economici, sociali e culturali. Ne consegue dunque che, mentre la competenza del Comitato è obbligatoria

V. Turp, Daniel, "Le contrôle du respect du Pacte international relatif aux droits économiques, sociaux et culturels”, in Mélanges MichelVirally, France, Paris-Pedone, 1991, pp. 468 y ss.; Alston, Philip, "Establishing a Right to Petition Under the Covenant on Economic, Social and Cultural Rights", in Collected Courses of the Academy of European Law, Holland, Martinus Nijhoff- Dordrecht-Boston-London, 1995.

8 In argomento v. amplius Malinverni, Giorgio, "Il Comitato dei diritti economici, sociali e culturali”, in Bestagno, Francesco (a cura di), I diritti economici, sociali e culturali, cit., pp. 184 y ss. 
per i rapporti periodici, essa è solamente facoltativa per le procedure contenziose.

\section{Cenni ai metodi interpretativi previsti negli artt. 31-33 della Convenzione diVienna del 1969}

La Convenzione di Vienna del 1969 sul diritto dei trattati (art. 31, par. 1) prevede che qualsiasi accordo debba essere interpretato in buona fede, sulla base del significato riconosciuto ai termini in esso previsti nel loro contesto ed alla luce dell'oggetto e scopo. ${ }^{9}$ Quanto al richiamo dello scopo e dell'oggetto dell'accordo è necessario ricordare che la finalità del metodo teleologico d'interpretazione non corrisponde alla rilevanza da esso assunta nella dottrina e specialmente nella prassi antecedente la Convenzione di Vienna. ${ }^{10}$ Brevemente può rammentarsi che, nella prassi relativa al periodo antecedente la Convenzione di Vienna, la considerazione della finalità di un accordo veniva normalmente effettuata per indirizzare a priori gli interpreti verso l'adozione o viceversa l'esclusione di una certa interpretazione mentre, in altri casi, soltanto per confermare la validità di una precedente interpretazione ottenuta tramite il ricorso ad altri strumenti esegetici. ${ }^{11}$ La Convenzione di Vienna tuttavia sembra avere sminuito la rilevanza dell'interpretazione finalistica, equiparando l'esame degli scopi dell'accordo a quella degli altri metodi intepretativi indicati nell'art. $31 .{ }^{12}$

Quanto al contesto del trattato la Convenzione di Vienna (art. 31, par. 2) prevede che esso è costituito dal preambolo, dagli eventuali allegati, da qualsiasi accordo intercorso tra tutte le parti contraenti in occasione della conclusione del trattato principale nonché da qualsiasi

9 In argomento v. Gardiner, Richard, Treaty Interpretation, United Kingdom, Oxford-OUP, 2008, pp. 152 y ss.

10 Sul punto v. Pustorino, Pietro, L'interpretazione della Convenzione europea dei diritti dell'uomo nella prassi della Commissione e della Corte di Strasburgo, Napoli, 1996, pp. 11 y ss.

11 Ibidem, pp. 12 y ss. V. altresì Kolb, Robert, Interprétation et création du droit international: esquisses d'une herméneutique juridique moderne pour le droit international public, Belgium, BruxellesBruylant, 2006, pp. 10 y ss.

12 V. ex multis Strozzi, Girolamo, Il diritto dei trattati, Italia, Torino-Giappichelli editore, 1999 , pp. 53 y ss. 
ulteriore strumento normativo posto in essere da una o più parti ed accettato dalle altre come riguardante il trattato. A proposito degli altri metodi interpretativi, la Convenzione di Vienna prevede che occorre tenere conto degli accordi successivi conclusi fra le parti in materia di interpretazione o applicazione delle disposizioni del trattato principale, della prassi applicativa di siffatto trattato nella misura in cui dalla medesima sia desumibile l'accordo delle parti sull' esegesi del trattato. Nella disciplina prevista nella Convenzione di Vienna si stabilisce altresì la possibilità (art. 32) di utilizzare altri mezzi sussidiari d'interpretazione degli accordi internazionali, in particolare le circostanze riguardanti la conclusione dell' accordo ed i lavori preparatori.

\section{L'INTERPRETAZIONE DEL PATTO SUI DIRITTI ECONOMICI, SOCIALI E CULTURALI DA PARTE DEL COMITATO NELLA TEORIA}

\section{Critica alla tesi dell'obbligatorietà per il Comitato degli} artt.31-33 della Convenzione di Vienna.

L'utilizzazione delle regole ermeneutiche di cui agli artt. 31-33 della Convenzione di Vienna da parte del CESCR sarebbe indispensabile all'efficacia dell'attività di "guardianship" del Patto esercitata dal Comitato. ${ }^{13}$ In caso contrario sarebbe obiettivamente arduo - così come è invece sia auspicabile sia nella stessa logica del controllo esterno esercitato dal Comitato - il consolidamento di una "jurisprudence costante" multi-livello in materia di protezione dei diritti socio-economici e culturali. ${ }^{14}$ Orbene, sempre secondo i sostenitori di questa tesi, anche a volere contestare la veridicità di quanto appena richiamato, è comunque difficile disconoscere che il rispetto di siffatte regole ermeneutiche è imposto al CESCR dal diritto internazionale generale. Esso, infatti, sarebbe suggerito almeno dalla circostanza che il CESCR, al pari degli altri comitati delle Nazioni Unite sui diritti umani, interpreta i singoli

13 V. per tutti Mechlem, Kerstin, "Treaty Bodies and the Interpretation of Human Rights", Vanderbilt Journal of Transnational Law, 2009, pp. 919 y ss.

14 Cosi Mechlem, Kerstin, op. ult. cit., pp. 919 y ss. 
diritti e le obbligazioni in materia di protezione dei diritti dell'uomo sovente al posto degli Stati sovrani (che sono vincolati, come è noto, al rispetto degli artt. 31-33 della Convenzione di Vienna). ${ }^{15}$

A nostro parere, queste conclusioni, sebbene ancorate a presupposti corretti, non risultano convincenti. E', infatti, eccessivo sostenere che dalla prima circostanza sopra indicata - e cioè dal ruolo "vicario" degli Stati contraenti svolto dal CESCR in materia di interpretazione del Patto - possa desumersi la necessità per quest'ultimo di conformarsi in ogni caso agli artt. 31-33 della Convenzione di Vienna. Ciò anche perché — almeno se si accoglie la tesi sostenuta dallo stesso Comitato sull'imputabilità delle obbligazioni derivanti dal Patto anche ad attori non statali (cd "non state actors") — ${ }^{16}$ può risultare arduo descrivere il ruolo del Comitato come sussidiario rispetto a quello degli Stati contraenti. ${ }^{17}$ Alcune considerazioni parzialmente differenti devono essere fatte a proposito della seconda circostanza sopra richiamata. In questo caso, infatti, ci pare persuasivo il ragionamento di chi sostiene che dall'osservanza degli artt. 31-33 della Convenzione di Vienna le decisioni del CESCR (in particolare le cd. "General Observations" ed i "General Comments") possono trarre autorevolezza e quindi possibilità di “compliance" da parte dei loro destinatari ${ }^{18}$. Resta peraltro non dimo-

$15 \mathrm{~V}$. in particolare Ksentini Ouhachi, Fatma Zohra, Les procédures onusienne de protection des droits de l'homme, Paris, 1994, pp. 73 y ss, anche per ulteriori riferimenti bibliografici.

16 V. di recente il General Comment n. 14 sul diritto alla salute (art. 12), par. 42, in cui il Comitato —mediante l'affermazione che: "While only States are parties to the Covenant and thus ultimately accountable for compliance with it" - perviene implicitamente a teorizzare una diversità di ruoli degli Stati contraenti e delle organizzazioni non-governative nell'attuazione dei diritti socio-economici e culturali contenuti nel Patto laddove sostiene che: "all members of society —individuals, including health professionals, families, local communities, intergovernmental and non-governmental organizations, civil society organizations, as well as the private business sector - have responsibilities regarding the realization of the right to health. State parties should therefore provide an environment which facilitates the discharge of these responsibilities".

17 Ciò anche perché una siffatta ricostruzione teorica indebitamente prescinderebbe dalla circostanza che nelle sopra menzionate osservazioni finali sull'Iran si è ammesso che la responsabilità dello Stato si estende anche agli atti imputabili ad organizzazioni non governative sui cui il medesimo eserciti un controllo effettivo.

18 Incidentalmente, può rilevarsi che tali difficoltà sarebbero invece eliminate se prevalesse, così come a noi sembra opportuno, un'interpretazione letterale delle norme pattizie, la quale suggerisce l'obbligatorietà del Patto soltanto per gli Stati contraenti. 
strato se siffatta autorevolezza e possibilità di "compliance" non possano che ottenersi tramite l'osservanza delle predette disposizioni. In senso contrario, ci sembrerebbero deporre le esperienze di taluni sistemi di garanzia operanti nell' ordinamento giuridico internazionale in contesti diversi da quello della tutela dei diritti socio-economici nel Patto. Ci riferiamo, ad esempio, all' esperienza del panel di ispezione della Banca Mondiale $^{19}$ ed quelle analoghe dei corrispondenti meccanismi di "accountability" operanti presso le principali banche internazionali di sviluppo a carattere regionale. ${ }^{20}$ In questi contesti infatti è agevole riscontrare esempi di decisioni prive di forza giuridica sul piano formale ma provviste di efficacia ed autorevolezza nella pratica nonostante la loro (talvolta parziale) inosservanza dei criteri interpretativi codificati nella Convenzione di Vienna. ${ }^{21}$ Sempre in senso contrario a quanto richiamato sopra, può ricordarsi il (quasi) unanime riconoscimento da parte degli Stati contraenti della rilevanza giuridica dei General Comments a prescindere da qualsiasi preventivo accertamento di un loro rispetto degli artt. 31-33 della Convenzione di Vienna. ${ }^{22}$ Da qui l'opportunità ma non anche l'obbligatorietà per il Comitato del ricorso ad interpretazioni del Patto sempre motivate e/o fondate su almeno uno dei criteri ermeneutici previsti nella Convenzione di Vienna. ${ }^{23}$ Siffatta opportunità non deve comunque ostare né all'eventuale elaborazione di interpretazioni evolutive del Patto né, per utilizzare un linguaggio caro alla Corte di Strasburgo, di interpretazioni ispirate alle esigenze di attualizzazione

19 In argomento ci sia consentito rinviare al nostro Il Panel di Ispezione della Banca Mondiale, Italia, Torino-Giappichelli editore, 2008, pp. 283 y ss.

20 V. sul meccanismo di accountability della Banca Europea di Ricostruzione e Sviluppo il nostro "In Search of New Ways to Ensure Effective Compliance with Environmental Procedures and Policies: the Experience of the European Bank for Reconstuction and Development with its Internal Recourse Mechanism”, in Treves, Tullio, [et al.], Non-compliance Procedures and Mechanisms and the Effectiveness of International Environmental Agreements, Holland, The HagueKluwer Law International, 2009, pp. 337 y ss.

21 Amplius si veda ancora il nostro Il Panel di Ispezione della Banca Mondiale, cit., pp. 283 y ss.

22 Quest'ultimo punto è stato lucidamente colto anche da Mechlem, Kerstin, "Treaty Bodies", cit., pp. 932 y ss.

23 V. infra par. 2.2. 
degli obblighi pattizi in capo agli Stati contraenti in materia di diritti sociali, economici e culturali. ${ }^{24}$

\section{Le principali difficoltà derivanti dall'interpretazione del Patto da parte del Comitato conformemente agli artt.31-33 della Convenzione di Vienna}

Rimane da chiarire l'esistenza di eventuali impedimenti all' utilizzazione dei criteri ermeneutici previsti nella Convenzione di Vienna da parte del Comitato.

Innanzitutto, è opportuno sottolineare che talune difficoltà all'utilizzazione delle regole interpretative della Convenzione di Vienna non possono desumersi dalla natura del CESCR. Quest'ultimo infatti - essendo un organismo di garanzia a carattere giuridico e non politico- è almeno astrattamente idoneo a ricorrere alla Convenzione di Vienna ai fini dell'interpretazione del Patto sui diritti economici, sociali e culturali. Siffatta idoneità non deve peraltro essere enfatizzata, nel senso che essa non può fare dimenticare che il Comitato è composto anche da individui privi di competenze tecnico-giuridiche. Sicché è palese che agli artt. 31-33 della Convenzione di Vienna il Comitato non è obbligato a ricorrere alla stessa stregua degli altri organismi di garanzia a carattere giuridico ed composti solamente da operatori del diritto. Quanto osservato, almeno secondo noi, non è contraddetto dalla fiducia degli organi giurisdizionali degli Stati contraenti verso il Comitato, manifestata mediante il frequente "allineamento" dell'interpretazione di singole libertà

24 Sulla funzione attualizzatrice dei valori fondamentali delle collettività degli Stati contraenti svolta dalla Corte di Strasburgo v. ex multis Letsas, George, A theory of interpretation of the European Convention on Human Rights, United Kingdom, Oxford-OUP, 2007, pp. 30 y ss; Prebensen, Soren, "Evolutive interpretation of the European Convention on Human Rights", in Mahoney, Paul, Matscher, Franz, Petzold, Hans, Wildhaber Luzius (eds), Protection des droits de l'homme: la perspective européenne, mélanges à la mémoire de Rolv Ryssdal = Protecting human rights: the european perspective, studies in memory of Rolv Ryssdal, Germany, Berlin-Bonn-München, Verlag, 2000, pp. 1123-1137; Focarelli, Carlo, Lezioni di diritto internazionale, t. I, Italia, PadovaCedam, 2008, pp. 153 y ss; Id, Equo processo e Convenzione europea dei diritti dell'uomo. Contributo alla determinazione dell'ambito di applicazione dell'art. 6 della Convenzione, Italia, Padova-Cedam, 2001, pp. 397- 405; Mosler, Henry, "Problems of interpretation in the case law of the European Court of Human Rights", in Essays on the development of the international legal order: in memory of Haro F. van Panhuys, Alphen aan den Rijn [etc.], 1980, pp. 149 y ss. 
e/o diritti socio-economici a quella fornita dal CESCR in particolare e, più in generale, alle interpretazioni accolte dai competenti comitati delle Nazioni Unite sui diritti umani nei rispettivi settori di competenza. ${ }^{25}$

In secondo luogo, è lecito domandarsi se un ricorso ai criteri della Convenzione di Vienna è consentito nel contesto delle Concluding Observations del Comitato. Al riguardo, è stata affermata in dottrina la compatibilità tra questi strumenti operativi e gli artt. 31-33 della Convenzione di Vienna. ${ }^{26}$ Tale tesi è stata argomentata richiamando l'atteggiamento positivo rispetto alle Concluding Observations in particolare delle ONGs. ${ }^{27}$ Queste ultime, infatti, sono state spesso inclini a considerare siffatti strumenti alla stregua di decisioni giudiziarie. Sebbene brillantemente argomentata tale opinione non ci pare condivisibile, almeno se essa viene intesa nel senso radicale che agli artt. 31-33 della Convenzione deve farsi riferimento nelle "Concluding Observations" del Comitato. Ciò perché differente è la funzione economico-sociale di questi strumenti operativi rispetto a quella delle decisioni delle corti regionali sui diritti umani. Come è noto, infatti, il principale obiettivo delle Concluding Observations non è il giudizio sull'operato degli Stati membri - come è il caso delle decisioni giudiziarie - ma l'indicazione, a mero titolo di suggerimenti ("suggestions"), di soluzioni e possibili rimedi ai fini di un'ottimale attuazione delle corrispondenti disposizioni pattizie negli ordinamenti giuridici dei Paesi membri. In terzo luogo, occorre chiedersi se sussistano impedimenti all'utilizzazione dei criteri interpretativi della Convenzione di Vienna nelle decisioni rese dal CESCR sui ricorsi individuali. In argomento ci sembra convincente l'opinione di chi ha sostenuto l'assenza di impedimenti ad un richiamo degli artt. 31-33 della Convenzione. ${ }^{28}$ Un siffatto richiamo infatti, pur non essendo obbligatorio è in ogni caso opportuno, anche in considerazione della funzione "quasi-giurisdizionale" svolta dal Comitato, a seguito dell'entrata in vigore del Protocollo addizionale sui ricorsi inter-individuali mediante le suddette pronunce. ${ }^{29}$

25 Cfr. Mechlem, Kerstin, "Treaty Bodies”, cit., pp. 920 e n. 67.

26 Ibidem, pp. 921 y ss.

27 Ibidem, p. 924.

28 Ibidem, pp. 920 y ss.

29 Ibidem, p. 926. 
Quanto fin qui evidenziato ci sembra riferibile anche a siffatti strumenti operativi. Questi ultimi — prevedendo delle guidelines per l'interpretazione del Patto destinate agli Stati contraenti - sicuramente possono fare ricorso ai criteri interpretativi della Convenzione di Vienna. Tuttavia ci pare egualmente consentito che, almeno in qualche occasione, il Comitato proceda a interpretazioni delle norme pattizie in deroga agli artt. 31-33 della Convenzione di Vienna. ${ }^{30}$ Una conferma di quanto sostenuto si ricava sia dal carattere accademico dei General Comments sia dalla circostanza che anche essi costituiscono l'espressione dell'attività svolta da soggetti, taluni componenti del CESCR, privi di un background giuridico. Nondimeno è da riconoscersi che —allontanandosi dagli artt. 31-33 della Convenzione di Vienna - esiste sempre il rischio che il Comitato finisca con l'appropriarsi di funzioni indebite nel senso cioè di non previste nella sua risoluzione istitutiva come, ad esempio, quella di "law making" e/o addirittura di funzioni contrarie a quella di garanzia del Patto per cui il CESCR è stato formalmente costituito. ${ }^{31}$ Trattasi, in ogni caso, di esiti contrastabili mediante un'attività preventiva di "self restraint" da parte del CESCR finalizzata ad accertare in concreto gli effetti derivanti dalle deroghe agli artt. 31-33 della Convenzione di Vienna. Quanto osservato non vale però a disconoscere anche l'idoneità del Comitato a promuovere, per utilizzare la terminologia dell'art. 13 della Carta ONU, uno "sviluppo progressivo" della tutela internazionale (ivi inclusa quella di diritto internazionale consuetudinario) dei diritti economici, sociali e culturali. ${ }^{32}$ In caso contrario, quale senso avrebbero la composizione ibrida (giuristi e non giuristi) del Comitato, la natura accademica dei General Comments e la discrezionalità del CESCR sui contenuti delle "Concluding Observations"?

$30 \quad$ Ibidem, p. 927.

31 Si pensi, a questo proposito, all'estrema labilità di confini tra l'interpretazione evolutiva (sicuramente ammissibile) e l'attività creativa o di "law making" (confliggente invece con gli artt. 31-33 della Convenzione di Vienna). In argomento da ultimo v. Corten, Olivier, "Les techniques reproduites aux articles 31 à 33 des conventions de Vienne: approache objectiviste ou approche volontariste de l'interpretation ? ", RGDIP, 2011, pp. 351 y ss.

32 E' appena il caso di sottolineare che la promozione di uno sviluppo progressivo delle garanzie in materia di tutela dei diritti economici, sociali e culturali è legittimo purché rispettoso sia degli obiettivi e della complessiva struttura del Patto sia della distinzione tra obblighi pattizi (“obligations”) e mere raccomandazioni (“desirable policy options”). 


\section{L'INTERPRETAZIONE DEL PATTO SUI DIRITTI ECONOMICI, SOCIALI E CULTURALI DA PARTE DEL COMITATO NELLA PRASSI}

\section{Cenni ai principali orientamenti interpretativi desumibili dalla "giurisprudenza" del Comitato}

E' opportuno adesso esaminare la "giurisprudenza" del Comitato al duplice scopo di enucleare i principi e prima ancora le tendenze generali emergenti dalla copiosa prassi (essenzialmente costituita dalle General Observations e dai General Comments) e di individuare così le varie tecniche ermeneutiche da esso utilizzate.

Un rilievo tuttavia si impone in via preliminare. Esso attiene alla cautela cui è ispirata, almeno nelle sue linee fondamentali, la predetta "giurisprudenza" così come è attestato anche dagli scritti di quegli autori che periodicamente hanno annotato e commentato i più importanti "General Comments" del CESCR, al pari dei corrispondenti strumenti di altri comitati delle Nazioni Unite sui diritti dell'uomo. Va ulteriormente precisato che la cautela dei membri (giuristi e non giuristi) del CESCR si è finora manifestata mediante comportamenti che solitamente prescindono da dichiarazioni di principio, da valutazioni teoriche approfondite dei singoli problemi, da posizioni nette suscettibili di impegnare definitivamente il Comitato in un senso oppure nell'altro. In effetti, sarebbe abbastanza inutile cercare nei vari General Comments e nelle Concluding Observations, anche in quelli più recenti e/o meglio strutturati, l'adesione a taluni orientamenti interpretativi perché maggiormente conformi alla ratio delle singole disposizioni pattizie. Né, d'altra parte, un tale atteggiamento ci sembra facilmente desumibile da un'analisi complessiva della “giurisprudenza”, la quale rivela sia un'ampia utilizzazione dei princìi sia l'accoglimento di orientamenti differenti, il cui impiego sembra suggerito (in prevalenza) dalle caratteristiche della situazione specifica ed in funzione della soluzione prescelta. Varietà di approcci e prudenza nelle dichiarazioni di principio risultano quindi le più sicure costanti della prassi del CESCR, i cui risultati assai male si prestano ad una rapida caratterizzazione a senso unico. Ciò è stato reso particolarmente evidente da alcune indagini su questa "giu- 
risprudenza", le quali, pur condotte in maniera abbastanza esauriente e con sguardo retrospettivo, rivelano appunto l'impossibilità pratica di desumere delle conclusioni univoche e pertanto si fermano ad una descrizione delle sue linee direttrici. ${ }^{33}$

Troppe volte invece la dottrina che si è occupata del Comitato e della sua prassi rivela una malintesa aspirazione a colmare il divario esistente tra le possibilità potenziali (e talvolta solamente desiderate) dal Comitato e la sua azione mediante una superflua sopravvalutazione di quest'ultima, effettuata con l'accentuazione del valore di singole statuizioni, talvolta nemmeno riprese nella prassi successiva. Ci sembrano esemplari al riguardo i General Comments nn. 8 e 13. Nel primo di essi, concernente le "Sanzioni”, il Comitato ha sostenuto l'esistenza di obbligazioni in capo a organizzazioni internazionali direttamente derivanti dal Patto. ${ }^{34} \mathrm{Nel}$ secondo, riguardante il diritto all' educazione, ${ }^{35}$ esso, al contrario, si è espresso, così come anche nel successivo General Comment n. 19 concernente il "diritto alla sicurezza sociale", in senso nettamente contrario all' esistenza di obbligazioni pattizie in capo ad entità diverse dagli Stati contraenti. ${ }^{36}$ E su siffatte singole statuizioni finali, poi, si è tentato di procedere a ricostruzioni teoriche organiche in ordine ai principi ed agli orientamenti di fondo accolti dal Comitato, per ricavarne conseguenze di ordine generale rispetto al complessivo valore della "giurisprudenza" ${ }^{37}$ Fatte salve queste premesse, non si vuole certo contestare la necessità di ancorare qualsiasi costruzione teorica ai dati concreti della prassi. E' evidente infatti che un'impostazione teorica può ammettersi come legittima soltanto se e nella misura in cui essa non si traduca in un tentativo di costringere entro rigidi schemi teorici una prassi che, come appunto quella del CESCR, facilmente sfugge ad una opera classificatoria. ${ }^{38}$

33 Cfr. Sodini, Raphael, Le Comité des droits économiques et sociaux et culturels, France, ParisMontchrestien, 2000, pp. 20 y ss.

34 CESCR, General Comment n. 8, del 12 dicembre 1997, E/C.12/1997/8, par. 11.

35 CESCR, General Comment n. 11, cit., parr. 3 e 9.

36 CESCR, General Comment n. 19, cit., par. 40 y ss.

37 Cfr. Sodini, Raphael, op. loc. cit., pp. 30 y ss.

38 Cfr. Odello, Marco, Seatzu, Francesco, Ensuring and Enforcing Economic, Social and Cultural Rights, cit., cap. IV. 
Tenendo presenti tali elementi è possibile procedere all'apprezzamento dei dibattiti sviluppatisi in ambito dottrinale sui particolari orientamenti e principi applicativi che il CESCR ha accolto, o comunque avrebbe dovuto accogliere, nell' esercizio della sua attività.

\section{Principi e metodologie utilizzati dal Comitato}

L'esame delle modalità utilizzate dal CESCR nella sua "giurisprudenza" per assolvere i compiti attribuitigli dalla risoluzione istitutiva non può essere disgiunto da un'attenta considerazione delle tecniche e dei principi ermeneutici elaborati a tale fine. Le circostanze sopra richiamate - soprattutto il fatto che gli approcci esegetici siano stati elaborati dal Comitato (quasi sempre) in maniera "pretoria" — hanno inevitabilmente inciso sulle caratteristiche di siffatta "giurisprudenza" anche se non sembrano avere prodotto conseguenze pratiche apprezzabili. Quelle più rilevanti possono essere raggruppate, a meri fini descrittivi, rispettivamente nei seguenti due punti:

a) Una parziale originalità metodologica che differenzia, per taluni aspetti, l'attività esegetica del CESCR rispetto a quelle corrispondenti dei sistemi/organismi di garanzia operanti presso le principali organizzazioni internazionali;

b) La dimensione evolutiva della prassi del Comitato in relazione al progressivo mutamento delle dinamiche socio-economiche sottese ai rapporti tra i privati e le autorità statali degli Stati contraenti del Patto.

E' appena il caso di sottolineare che le predette peculiarità sono riscontrabili soltanto in principio, poiché evidentemente non è sempre facile per il CESCR procedere per rigide alternative trascurando in particolare la discrezionalità di cui godono i singoli membri che lo compongono. Questo è vero soprattutto perché il CESCR è un organismo di garanzia che — sebbene non beneficiario di una libertà incondizionata nell' esercizio della sua attività interpretativa operante rispetto ad insiemi normativi scritti ed omogenei (leggasi: le disposizioni del Pat- 
to e dell'allegato Protocollo Opzionale sui ricorsi individuali) nonché funzionalmente preordinati alla realizzazione di un disegno unitariorisulta comunque ispirato nel suo concreto modus operandi a valutazioni orientate all'armonia dell'azione interpretativa. Una conferma si ricava dalle affermazioni, contenute nel General Comment n. 9 sull' applicazione interna del Patto, nella parte in cui testualmente si osserva che: «Questions relating to the domestic application of the Covenant must be considered in the light of two principles of international law (i.e. Article 27 of the Vienna Convention on the Law of Treaties and Article 8 of the Universal Declaration of Human Rights» ${ }^{39} \mathrm{e}$, ancora di più, in quelle, previste nel "General Comment" n. 13 sul diritto all' educazione, laddove si stabilisce che: «the Committee takes the view that States parties are required to ensure that education conforms to the aims and objectives identified in article 13 (1), as interpreted in the light of the World Declaration on Education for All (Jomtien, Thailand, 1990) (art. 1), the Convention on the Rights of the Child (art. 29 (1)), the Vienna Declaration and Programme of Action (Part I, para. 33 and Part II, para. 80), and the Plan of Action for the United Nations Decade for Human Rights Education». ${ }^{40}$

A prescindere da quanto esplicitato nei due General Comments appena richiamati ovviamente l'azione interpretativa del CESCR acquista rilievo specialmente nei casi di incertezze sul significato pratico-operativo di una o più disposizioni pattizie. Nelle suddette ipotesi, infatti, è palese che i membri del CESCR possano intervenire mediante ricorso (discrezionale) ad una vasta serie di strumenti logico-concettuali finalizzati alla risoluzione dei dubbi interpretativi. In particolare, va segnalato che il Comitato ha fatto spesso richiamo agli obiettivi delle singole disposizioni e/o dell'intero Patto: e cioè a tutti quegli strumenti interpretativi che consentono di attribuire alle norme convenzionali un significato suscettibile di coordinamento con il complesso delle valutazioni in cui le medesime si trovano inserite e nel contempo di attribuire loro il massimo della funzionalità. Al riguardo, basti ricordare che il CESCR nel General Comment n. 5 sui diritti delle persone disabili — dopo avere preliminarmente osservato che: «The Covenant does not refer explicitly to

39 CESCR General comment n. 9, cit., par. 3.

40 CESCR General comment n. 13, cit., par. 2. 
persons with disabilities»— ha stabilito mediante un implicito ricorso al metodo teleologico che: «the Covenant's provisions apply fully to all members of society, persons with disabilities are clearly entitled to the full range of rights recognized in the Covenant» ${ }^{41}$.

\section{A. Il principio di economia giurisprudenziale quale criterio estraneo alla "giurisprudenza" del CESCR}

I cenni fatti finora agli orientamenti generali della "giurisprudenza" del Comitato non sarebbero completi senza un riferimento alla portata ed ai limiti del principio di economia giurisprudenziale. A questo proposito, innanzitutto, preme sottolineare che il CESCR - diversamente da altri organismi di garanzia a carattere giurisdizionale come, ad esempio, la Corte europea dei diritti dell'uomo - ha omesso di muoversi nella direzione di scongiurare pronunce ripetitive in ossequio al principio di "economia giurisprudenziale". Basti ricordare, a titolo esemplificativo, il carattere quasi seriale di numerose Concluding Observations, evidenziato sia dal fatto che esse rivestono la medesima struttura formale (il riferimento è in particolare alla bi-partizione tra "Factors and difficulties impeding the implementation of the Covenant" e "Positive Aspects") sia dal costante ricorso alle stesse tipologie di censure spesso formulate secondo terminologie lessicali quasi identiche tra $l_{0 r o}{ }^{42}$. In altri termini, il CESCR non ha mai ritenuto di doversi pronunciare su ciò che esso stesso ha ritenuto fondamentale ai fini dell'esercizio della sua funzione di garanzia dei diritti tutelati dal Patto, intervenendo esclusivamente nelle ipotesi in cui tale economia di giudizio rischiava di tradursi in una mancanza di "dialogo" con i Paesi contraenti impegnati nell'attuazione del Patto. Al contrario, esso ha scelto di intervenire, conformemente al proprio mandato istitutivo e con regolarità, nella dialettica aperta dalla presentazione dei rapporti statali mediante la presentazione di osserva-

${ }^{41}$ CESCR, General comment n. 5, cit., par. 5. In argomento v. Seatzu, Francesco, "The UN Committee on Economic, Social and Cultural Rights and Disability: General Comment No. 5 and Beyond", Diritti Umani e Diritto Internazionale, 2011, pp. 356 y ss.

42 Si compari, a mero titolo esemplificativo, UN Doc. E/C. 12/BEL/CO/3, 4 Gennaio 2008, par. 38 e UN Doc. E/C.12/1/Add.105, 1 Dicembre 2004, par. 55. Amplius Odello, Marco, Seatzu, Francesco, Ensuring and Enforcing Economic, Social and Cultural Rights, cit., cap. IV. 
zioni dettagliate ed esaustive sulle singole questioni e/o problematiche connesse all'attuazione dei diritti socio-economici nei Paesi autori dei rapporti periodici. ${ }^{43}$

L'estraneità del principio dell'economia giurisprudenziale al modus operandi del CESCR — si giustifica, a nostro avviso, se si pensa, oltre al mandato istitutivo del Comitato, alla "filosofia" dei rapporti degli Stati contraenti e alla loro finalità di fornire agli Stati autori dei rapporti raccomandazioni affinché essi rispettino e attuino i loro obblighi pattizi. Ancora, tale circostanza si comprende se si pensa al carattere necessitato, perché interlocutorio, delle osservazioni finali, comprovato in particolare dal fatto che il Comitato può adottare questi atti persino in assenza di un rapporto statale. ${ }^{44}$ Quanto appena sottolineato peraltro non deve essere inteso nel senso che la risoluzione istitutiva del CESCR ha attribuito ai componenti di questo organismo di controllo un potere discrezionale illimitato nella gestione e nel successivo "follow up" dei rapporti periodici degli Stati contraenti. Una siffatta conclusione infatti sarebbe sicuramente errata, anche perché smentita dal sopra indicato carattere quasi standardizzato — come si è detto- delle Concluding Observations: carattere questo che si pone eminentemente a garanzia ed a beneficio degli stessi Stati contraenti. Ne consegue pertanto che le attività del CESCR di revisione e "follow up" dei rapporti statali incontrano un limite nel (sia pure non formalmente previsto) obbligo per il Comitato di esame delle singole argomentazioni dedotte nei rapporti periodici. Ciò poiché è evidente che tralasciare da parte del Comitato anche soltanto alcune argomentazioni utilizzate dagli Stati nei loro rapporti a dimostrazione dell' effettiva "compliance" agli obblighi pattizi non è un approccio sempre riconducibile all' uso ma piuttosto all'abuso del sopra richiamato principio di economia giurisprudenziale.

43 Odello, Marco, Seatzu, Francesco, op. ult. cit., cap. IV.

${ }^{44}$ Ciò si evince, sia pure indirettamente, dalla «Rule 59-Non submission of reports» del cd. statuto del CESCR ("Rules of Procedure of the Committee") la quale, al par. 2, testualmente prevede: "If, after the reminder referred to in paragraph 1 of this rule, the State party does not submit the report required under rule 58 of these rules, the Committee shall so state in the annual report which it submits to the Council". Per il testo in lingua originale (inglese) dello statuto del Comitato v. il sito web ufficiale del CESCR, http://www2.ohchr. org/english/bodies/cescr/. 
B. Il ricorso alla buona fede come criterio interpretativo da parte del CESCR

La necessità di procedere ad una ricerca empirica della metodologia interpretativa maggiormente consona alle esigenze del "dialogo" instaurato dal Comitato con gli Stati contraenti singolarmente, nel caso delle Concluding Observations — ovvero nella loro generalità- nel caso dei General Comments, si ricava rispettivamente:

a) dalla circostanza che il sistema di garanzie del Patto è finalizzato - come anche la recente elaborazione del Protocollo facoltativo al Patto sui diritti economici, sociali e culturali attesta - alla tutela dei singoli, sia pure non sempre direttamente.

b) dal fatto, non meno significativo, che al CESCR, nel caso dell'accertamento di violazioni di uno o più diritti sanciti nel Patto da parte di uno Stato contraente, compete fornire puntuali indicazioni (sia pure non giuridicamente vincolanti) sulle misure da adottare per ripristinare la legalità.

Passando alla fisionomia assunta dalla ricerca, da parte del Comitato, della metodologia interpretativa più rispondente alle peculiarità del caso specifico (ovvero, a seconda delle circostanze, delle peculiarità della situazione in cui concretamente versa lo Stato autore del rapporto e/o responsabile della mancata proposizione del ricorso al CESCR, da una parte, e di quelle specificamente attinenti il singolo diritto o libertà da tutelare dall'altra parte) va precisato che essa ricerca è complicata da quanto osservato sopra a proposito della parziale adesione del Comitato ai criteri interpretativi previsti nella Convenzione di Vienna. E' evidente, infatti, che dai suddetti criteri, i quali nella loro globalità, valorizzano il fine perseguito dalla norma da interpretare, derivano condizionamenti al potere/libertà del Comitato di ricercare equitativamente le metodologie ermeneutiche rispondenti alle esigenze del caso specifico (leggasi: funzionali alla protezione dei singoli diritti garantiti nel Patto ed eventualmente oggetto di General Comments oppure, se del caso, dello Stato contraente sul cui rapporto periodico vertono le Concluding Observations elaborate dal CESCR). Pertanto non stupisce che il Comitato 
abbia fatto talvolta ricorso per l'interpretazione delle disposizioni del Patto - sia pure non sempre esplicitamente — al generale criterio della buona fede, il quale, nella sua innegabile duttilità, è sicuramente in grado di consentire l'utilizzo della metodologia interpretativa più consona al caso specifico, di cui si è già detto. Emblematico di siffatta attitudine è il General Comment n. 9 in cui il CESCR - dopo avere correttamente premesso che: «The International Covenant on Economic, Social and Cultural Rights contains no direct counterpart to article 2, paragraph 3 (b), of the International Covenant on Civil and Political Rights, which obligates States parties to, inter alia, "develop the possibilities of judicial remedy"»—— ${ }^{45}$ ha stabilito che: «Nevertheless, a State party seeking to justify its failure to provide any domestic legal remedies for violations of economic, social and cultural rights would need to show either that such remedies are not "appropriate means" within the terms of article 2, paragraph 1, of the International Covenant on Economic, Social and Cultural Rights or that, in view of the other means used, they are unnecessary»(corsivo aggiunto). ${ }^{46}$

\section{La specialità di natura oggettiva del Patto e i suoi effetti sul sistema pattizio}

Nei precedenti paragrafi si sono esaminati i principali strumenti ermeneutici ai quali si ricorre nella prassi del Comitato. Ciò al fine di evidenziare sia le principali divergenze sia le più significative affinità rispetto alla corrispondente disciplina prevista nella Convenzione di Vienna. Nel presente paragrafo è necessario soffermarsi invece sulla questione del fondamento normativo degli approcci esegetici "con caratteri originali" utilizzati dal CESCR. In particolare, occorre mettere in risalto gli elementi di specialità del sistema del Patto. A nostro parere, esso è caratterizzato sia da una specialità oggettiva, vale a dire relativa al contenuto ed agli obiettivi stabiliti nelle singole disposizioni pattizie, sia da una specialità riguardante l'esistenza di un meccanismo accentrato di con-

45 V. anche, tra i tanti, UN Doc. E/C. 12/DEU/CO/5, 20 Maggio 2011, par. 8; UN Doc.

E/C. 12/BRA/CO/2, 19 Giugno 2009, par. 22.

46 CESCR, General Comment n.14, par. 5. 
trollo sull'effettivo rispetto delle norme contenute nel Patto e nell'allegato Protocollo facoltativo. ${ }^{47}$

Per quanto attiene alla specialità oggettiva del Patto, è interessante sottolineare uno degli aspetti a cui, quasi costantemente, si fa riferimento nella prassi del CESCR: l'esistenza di obblighi "a realizzazione progressiva”. ${ }^{48} \mathrm{Al}$ riguardo, va sottolineato che tale profilo di specialità del Patto è spesso invocato dal Comitato per giustificare un'interpretazione finalistica di quelle disposizioni pattizie che —come, ad esempio, l'art. 2, par. 1 - prevedono siffatte obbligazioni giuridiche ${ }^{49}$. Ragionando sulla base di queste premesse è anche possibile ricavare una conferma dell'idoneità del metodo teleologico all'esegesi delle norme contenenti obblighi “a realizzazione progressiva”. Questo perché il contenuto non definibile a priori di siffatte obbligazioni —e, dunque, di difficile scrutinio da parte dei competenti organi internazionali di controllo - sembra prestarsi assai bene all'utilizzazione di tale criterio. Da qui la legittimità del ricorso al metodo teleologico per l'interpretazione di quelle norme pattizie che - come, ad esempio, l'art. 2, par. 1 del Patto - contengono obblighi a realizzazione non immediata.

Passando al secondo profilo di specialità —e cioè all' esistenza di un organismo indipendente di controllo, da un lato, e agli effetti giuridici riconducibili a tale peculiare conformazione del Patto- può dirsi che esso ha avuto dei riflessi importanti anche sulla "giurisprudenza" del Comitato. ${ }^{50}$ Perfettamente consapevole di tale specialità del Patto il CESCR si è complessivamente dimostrato all'altezza del ruolo di "vi-

47 Analoghe forme di specialità sono state riconosciute anche nel sistema della Convenzione europea dei diritti dell'uomo da Pustorino, Pietro, L'interpretazione della Convenzione Europea dei diritti dell'uomo, cit., pp. 145 y ss.

48 V. CESCR, General Comment n. 3, cit., par. 9, in cui si afferma che l'art. 2, par. 1 del Patto richiede allo Stato: "to move as expeditiously and effectively as possibile". In argomento v. ex multis Pisillo Mazzeschi, Riccardo, "Sulla natura degli obblighi internazionali di tutela e promozione dei diritti economici, sociali e culturali”, in Bestagno, Francesco (a cura di), I diritti economici, sociali e culturali, cit., pp. 15 y ss. e più ampiamente Pisillo Mazzeschi, Riccardo, "Responsabilité de l'état pour violation des obligations positives relatives aux droits de l'homme”, Recueil des cours, t. 333, 2008, pp. 180 y ss.

49 Cfr. Odello, Marco, Seatzu, Francesco, Ensuring and Enforcing Economic, Social and Cultural Rights , cit., cap. I, IV e V.

50 Ibidem, cap. IV. 
gilante" attribuitogli dalla sua risoluzione istitutiva. Al riguardo, occorre mettere in risalto, innanzitutto, che lo stesso Protocollo facoltativo non vieta, nei casi di rinuncia da parte del ricorrente individuale, che il Comitato possa decidere di proseguire comunque l'accertamento di violazioni del Patto, naturalmente sempre che esso però lo ritenga funzionale ad un'efficace protezione dei diritti economici, sociali o culturali. ${ }^{51}$ Analoghe conclusioni, almeno a nostro parere, possono ricavarsi dalla stessa risoluzione istitutiva del CESCR che, come già ricordato sopra, consente di procedere alla verifica di violazioni di norme pattizie anche a prescindere dalla formale presentazione di un rapporto periodico da parte dello Stato direttamente interessato dal procedimento monitorio. ${ }^{52}$ Facilitato dall'esistenza di un siffatta facoltà il Comitato ha, sia pure indirettamente, accreditato un principio di grande rilevanza pratica: e ciò̀ che anche le Concluding Observations (e non già soltanto i General Comments) possono servire a chiarire, garantire e ulteriormente sviluppare i contenuti delle norme convenzionali e quindi anche a promuovere il rispetto, da parte di tutti gli Stati contraenti, degli obblighi dai medesimi assunti. ${ }^{53}$

Sulla scorta delle osservazioni finora effettuate è possibile mettere in risalto alcune questioni che - sebbene concernenti il framework normativo-istituzionale del Patto sui diritti economici, sociali e culturali- agevolmente si prestano ad essere estese anche ad altri sistemi convenzionali inglobanti corrispondenti meccanismi internazionali di garanzia. In particolare, dalla prassi del CESCR è facilmente deducibile che l'istituzione di un siffatto organismo indipendente deputato a vigilare sul rispetto delle disposizioni pattizie comporta, da parte degli Stati contraenti sottoposti al suo controllo diretto, anche l'accettazione (implicita) di una "giurisprudenza" ovvero di una prassi a carattere evolu-

51 Complementare a questa disposizione è quella, prevista all'art. 4 del Protocollo facoltativo, che consente al Comitato di rifiutare l'esame di un ricorso se da esso non risulta evidente che: " .... the author has suffered a clear disadvantage, unless the Committee considers that the communication raises a serious issue of general importance”. In argomento v. Langford, Malcom, Closing the Gap?: an Introduction to the Optional Protocol to the International Covenant on Economic, Social and Cultural Rights, in Nordisk tidsskrift for menneskerettigheter, 2009, pp. 1 y ss.

52 Amplius Odello, Marco, Seatzu, Francesco, Ensuring and Enforcing Economic, Social and Cultural Rights , cit., cap. III.

53 Ibidem, cap. IV. 
tivo eventualmente estesa alle regole di diritto internazionale generale applicabili alla materia oggetto del $\mathrm{Patto}^{54}$. Il fondamento di quanto appena rilevato, a nostro parere, può individuarsi nella stessa volontà dei Paesi contraenti di riconoscere a taluni organi, da una parte, la facoltà di interpretare in maniera anche non vincolante le disposizioni pattizie in questione nonché, dall'altra parte, quella di indirizzare l'esatta applicazione delle disposizioni convenzionali all'interno degli ordinamenti giuridici nazionali. In altri termini, è legittimo affermare che nei sistemi pattizi contraddistinti dalla presenza di meccanismi di garanzia, anche a carattere non giurisdizionale, i Paesi contraenti rilascino automaticamente a questi ultimi una sorta di autorizzazione ad espletare un'attività di integrazione e sviluppo delle norme su cui concretamente è esercitato il controllo. Ciò, a nostro avviso, aiuta a comprendere e soprattutto giustifica le occasionali discrasie contenutistiche tra le norme pattizie e i relativi General Comments nonché le frequenti oscillazioni nella "giurisprudenza" del CESCR sul modo di intendere, ad esempio, le cd. obbligazioni essenziali (“core obligations”) del Patto.

\section{Cenni alla prassi di altri organismi di garanzia a carattere internazionale} in materia di protezione dei diritti economici e sociali. Il mancato recepimento delle linee di tendenza seguite dal Comitato

E' ora utile domandarsi se i risultati emersi dall' esame della "giurisprudenza” del CESCR trovino conferma, integralmente o parzialmente, nella prassi di altri meccanismi di garanzia operanti all'interno di sistemi normativi sulla tutela dei diritti umani contraddistinti da profili di specialità analoghi a quelli già riscontrati nel Patto e nell'allegato Protocollo facoltativo sui ricorsi individuali. Si tratta di comprendere in particolare se gli orientamenti della "giurisprudenza" del CESCR risultino seguiti anche nelle prassi di altri comitati delle Nazioni Unite sui diritti dell'uomo. Ciò eminentemente allo scopo di valutare l'idoneità

54 Così esattamente con riguardo al sistema della Cedu Pustorino, Pietro, L'interpretazione della Convenzione Europea dei diritti dell'uomo, cit., pp. 166 y ss. In argomento v. anche Garapon, Antoine, "Les limites à l'interpretation évolutive de la Convention européenne des droits de l'homme”, Revue trimestrielle des droits de l'homme, 2011, pp. 439 y ss.; Lucas-Aberni, Katia, Le revirement de jurisprudence de la Cour européenne des droits de l'homme, Bruxelles-Bruylant, 2008. 
della“giurisprudenza” del CESCR ad alterare le norme consuetudinarie codificate nella Convenzione di Vienna nel senso suggerito dal predetto Comitato.

Preliminarmente va sottolineato che l'atteggiamento abbastanza critico assunto dal CESCR nei confronti delle disposizioni sull'interpretazione dei trattati contenute nella Convenzione di Vienna (Artt. 31-33) - coerente, a nostro parere, con la posizione dello stesso Comitato contraria al recepimento del diritto internazionale generale per l'esegesi delle norme pattizie - non è comunque finalizzato alla chiusura di tale sistema normativo all' ordinamento internazionale ${ }^{55}$. Nondimeno resta il fatto che — diversamente, ad esempio, dalla giurisprudenza della Corte europea dei diritti umani - la "giurisprudenza" del CESCR è obiettivamente inidonea a contribuire alla genesi e/o allo sviluppo di norme generali in materia di interpretazione degli accordi internazionali. ${ }^{56}$

Quanto appena sottolineato trova una significativa, sia pure indiretta, conferma nella copiosa prassi riguardante il "gemello" Patto delle Nazioni Unite sui diritti civili e politici del $1966^{57}$. Come è stato osservato già da altri, dalla suddetta prassi è infatti agevole ricavare un'incidenza sostanzialmente trascurabile dei criteri ermeneutici e degli approcci interpretativi utilizzati dal CESCR. ${ }^{58} \mathrm{Ne}$ consegue pertanto che né le Concluding Observations né i General Comments del CESCR risultano at-

55 Cfr. Odello, Marco, Seatzu, Francesco, Ensuring and Enforcing Economic, Social and Cultural Rights, cit., cap. IV. Una volontà del CESCR di non chiudere il sistema del Patto all'ordinamento internazionale è deducibile anche da numerose "Concluding Observations" ( e.g. UN Doc. E/C. 12/I/Add. 103, 14 Dicembre 2004, par. 36; UN Doc. E/C.12/MEX/CO/4, 9 Giugno 2006, par. 34; UN Doc., E/C.12/CO/FRA/CO/3, par. 50).

56 Cfr. Pustorino, Pietro, L'interpretazione della Convenzione europea, cit., pp. 170 y ss.

57 Il Patto, adottato il 16 dicembre 1966, è entrato in vigore il 3 gennaio1976. In argomento v. ex multis Bouziri, Nejib, La protection des droits civils et politiques par l'O. N. U: l'ceuvre du Comité des Droits de l'Homme, France, Paris-Pedone, 2003; Joseph, Sara, Schultz, Jenny, Castan, The International Covenant on Civil and Political Rights: Cases, Materials and Commentary, Oxford - OUP, 2004; Kumar, Avinash, Civil and political rights as human rights: a study into the International Covenant, New Delhi - Serials Publications, 2003; Novak, Manfred, U. N. Covenant on Civil and Political Rights : CCPR commentary, Kehl [etc.] - Springer, 2005.

58 Cfr. Conte, Alex, Burchill, Richard, Defining Civil and Political Rights: The Jurisprudence of the United Nations Human Rights Committee, United Kingdom, Abingdon-Ashgate 2009, pp. 13 y ss. 
tualmente capaci di indirizzare le "giurisprudenze" degli altri comitati delle Nazioni Unite sui diritti dell'uomo verso le posizioni assunte e precedentemente sperimentate all'interno del Patto sui diritti economici, sociali e culturali. 